\title{
Audit and feedback to reduce inappropriate Full Blood Count pathology testing
}

\author{
Fergus William Gardiner* \\ Calvary Health Care, Australia
}

Received: November 13, 2015

Accepted: December 17, 2015 Online Published: December 23, 2015

DOI: $10.5430 /$ jha.v5n2p42

URL: http://dx.doi.org/10.5430/jha.v5n2p42

\begin{abstract}
Objective: This study was designed to reduce inappropriate Full Blood Count (FBC) pathology testing on specific hospital wards. It was hoped that by auditing requested Full Blood Counts, the researcher would be able to determine and benchmark appropriateness before conducting feedback interventions to promote appropriate pathology test ordering.

Methods: To reduce inappropriate Full Blood Count pathology test ordering, the researcher audited patient notes and pathology test request forms in June 2015 before conducting audit and feedback interventions in July, August, and September 2015 on the hospital ward areas. The feedback intervention consisted of auditing patient notes, pathology request forms, and the local pathology clinical integration systems to determine Full Blood Count appropriateness. This data was then communicated to the attending doctor and requesting doctor during feedback sessions. To conceptualize appropriate pathology test ordering, the researchers highlighted the "Framework for analysis of test ordering" during scheduled feedback sessions. It was hypothesized that audit and feedback would decrease the amount of inappropriate Full Blood Counts ordered.

Results: After receiving the audit and feedback intervention, clinicians were more likely not to order inappropriate Full Blood Counts $(64.60 \%$ vs. $23.40 \%)$, specifically providing adequate clinical reasoning for the test, $t(4.6706)=0.0429, p=.05$.

Conclusions: This study found that audit and feedback sessions significantly improved appropriate pathology test ordering and the clinical reasoning associated with Full Blood Counts.
\end{abstract}

Key Words: Medical education, Inappropriate pathology test ordering, Evidence-based pathology test ordering, Specimen error reduction, Over-ordered medical tests

\section{INTRODUCTION}

Pathology testing has an important role in the diagnosis, monitoring, and screening for disease. This reliance has led to dramatic increases in pathology testing in many countries. ${ }^{[1-3]}$ While some of this increase is appropriate, reflecting advances in training and technology, ${ }^{[4]}$ as detailed by Gardiner ${ }^{[5]}$ there is a growing trend of inappropriate/unnecessary pathology ordering. This includes inappropriate pathology requesting which are "performed at the wrong time or too frequently to be of value in diagnosis, prognosis, or ongoing clinical management". ${ }^{6]}$ Concerns have been raised on the appropriateness of commonly ordered tests, including Full Blood Counts (FBCs) ${ }^{[7]}$ Liver Function Tests (LFTs), ${ }^{[8]}$ Vitamin $\mathrm{B}_{12} /$ folate (Vit $\left.\mathrm{B}_{12}\right),{ }^{[9]}$ Thyroid Function Tests (TFTs), ${ }^{[10]}$ Vitamin D (Vit D), Prostate-Specific Antigen (PSA), and Troponin. ${ }^{[11]}$ Australian data suggests that many of the pathological testing does not necessarily meet recommended guidelines, including $25 \%-75 \%$ of tests ordered not being supported by evidence or expert opinion. ${ }^{[12]}$ As such, inappropriate pathology testing could be considered

*Correspondence: Fergus William Gardiner; Email: gus_gardiner@ hotmail.com; Address: Calvary Health Care, Australia. 
low-value in that it does not benefit the ongoing management of the patient. ${ }^{[13]}$

A number of influences have been described ${ }^{[14-17]}$ for the test ordering behaviors of clinicians, including doctor related factors such as experience, perceived medico-legal risk, patient related factors such as anxiety, hospital related factors such as business processes, systems related factors such as the development of new tests, health system incentives favoring more tests, and cultural beliefs that more is better. Inappropriate pathology testing can lead to over-diagnosis, which leads to unnecessary treatment, and adds to the risk of patient harm. Furthermore, inappropriate pathology leads to a reduction in the value of the pathology test, specifically reducing the patient benefit as it compares to expenditures. Judgments about value should be a key consideration in decision making, although in healthcare it can be lacking. ${ }^{[13]}$ High cost interventions may provide good value because they are highly beneficial; alternatively, low-cost interventions may have little or no value if they provide minimum benefit. ${ }^{[13]}$

Medical testing costs within Australia are increasing at an unsustainable rate. ${ }^{[11]}$ There has been debate around healthcare being rationed within Australia. ${ }^{[18,19]}$ However, the term rationing implicitly refers to restricting the use of any intervention, regardless of its benefit to the patient. ${ }^{[12]}$ It is the researcher's belief that the best way to avoid inadvertent reductions in effective medical testing is to identify and reduce wasteful practices, and demonstrate those medical tests which provide value. As such, the first step toward providing high-value care is to discontinue interventions that provide no benefit, including routine and clinically unjustified medical testing. This strategy would help make healthcare more sustainable while providing enhanced patient outcomes through clinicians carefully assessing the value of the medical intervention. The successful delivery of high-value care depends on developing evidence which will help administrators understand which services provide good value. ${ }^{[20]}$

FBCs at Calvary Hospital accounted for a significant amount of the tests ordered in 2013-2014 (14\%). Much of these FBC tests were deemed inappropriate, such as repeated FBC pathology testing (7.45\%). Previous research ${ }^{[5]}$ highlighted that the hospital ward areas had the highest percentage of inappropriate FBC pathology testing repeats, at an average inappropriate rate of $18 \%$. These repeated tests did not contribute to the ongoing management of the patient and could be deemed a low-value pathology test.

Recognizing this trend and to promote appropriate high-value pathology testing, the researcher implemented an audit and feedback intervention on the hospital wards targeting FBCs directly.

Hypothesis: Audit and feedback reduces inappropriate FBC pathology testing in targeted ward areas.

\section{METHOD}

\subsection{Case hospital}

The case hospital is an accredited 250 bed public hospital located in Canberra Australia. The hospital has many services including an Emergency Department, an Intensive and Coronary Care Unit, medical and surgical wards, a Maternity Unit, a voluntary psychiatric ward, and ambulatory care and outreach facilities and services. The hospital is a teaching hospital with associations with local universities. ${ }^{[21]}$ The fully accredited clinical laboratory provides specialist pathology services to the general public and while patients are in hospital. ${ }^{[22]}$

\subsection{Audit}

To reduce inappropriate FBCs pathology test ordering, the researcher audited patient notes and pathology request forms in June 2015 before conducting feedback interventions in July, August, and September 2015 on the hospital ward areas. This strategy allowed targeted intervention by department and clinician.

\subsection{Inappropriate pathology testing ordering criteria}

A FBC was deemed inappropriate if the test was ordered without adequate clinical reasoning, and using The National Coalition of Public Pathology (2012) definition of inappropriate pathology, "performed at the wrong time or too frequently to be of value in diagnosis, prognosis, or ongoing clinical management." ${ }^{[6]}$ Furthermore, if the results were not accessed in the hospital's clinical information systems or used in ongoing patient management within 24 hours, the test was deemed inappropriate. This information was gained by referencing the signature access data via the hospital's pathology clinical information system.

Inadequate clinical reasoning:

- Including results not accessed or used in the ongoing patient management within 24 hours.

Preformed at the wrong time or too frequently:

- Including repeated pathology testing without adequate clinical reasoning.

- Pathology request forms submitted and/or completed 24 hours before the specimen was collected. This did not include requests ordered 24 hours in advance for a justified clinical reason. If the pathology test was ordered in advanced, although was used in patient man- 
agement, it was deemed appropriate due to adequate clinical reasoning.

Each criterion was allocated a score of 1 . A score of 0 indicates compliance. The criteria were non-accumulative; if a FBC was both inadequately reasoned and performed at the wrong time or too frequently, only 1 point was received. To further validate the clinical reasoning of the FBC pathology test ordered, the researcher used the Royal College of Pathologists Australasia online manual. ${ }^{[23]}$

\subsection{Feedback}

The feedback intervention included formulating the previous days FBC pathology request forms in the hospital laboratory before accessing the clinical records and clinical information systems on the wards. The researcher determined appropriateness of the pathology test ordered, followed by direct education with the requesting and admitting doctor who ordered the test, followed by detailing the issues associated with inappropriate pathology test ordering. This allowed identification of unconscious incompetence, or potential unknowns or limitations of the treating clinician. ${ }^{[24]}$ These results were tabled at the conclusion of the intervention.

During feedback, the researcher highlighted the "Framework for analysis of test ordering" ${ }^{[11]}$ which included the following questions, relating to pathology test ordering:

- Why did you order the test?

- How will the ordered test alter your management?

- What are the potential risks of ordering or not ordering the test?

- Is there a potential of over diagnosis?

- What is the likelihood of a positive result?

- What is the prevalence of the provisional diagnosis?

- Were you influenced by anything else?

- Are there guidelines related to this presentation?

Researchers asked the clinician to answer this framework based on the last FBC pathology test ordered (not necessarily the test deemed inappropriate). The framework was beneficial in conceptualizing rational pathology test ordering.

\subsection{Statistical analysis}

A paired $t$-test was used to compare results from the prefeedback month of June 2015 to the feedback months of July, August, and September 2015. Means were compared throughout the intervention.

\section{Results}

\subsection{Audit and feedback}

A total of 300 patients were included in the study. The researcher audited patient notes and pathology request forms; 113 pre-intervention and 187 intervention. The preintervention month had $64.60 \%$ inappropriate FBC testing whereas the intervention months had an average of $32.15 \%$ inappropriate $\mathrm{FBC}$ testing. Of those, the pre-intervention month had $35.5 \%$ inadequately clinical reasoned, and $30.1 \%$ performed at the wrong time or too frequently. The intervention months had an average of $18.1 \%$ inadequately clinical reasoned, and $14.0 \%$ performed at the wrong time or too frequently. Of note, the pre-intervention month had $8.0 \%$ of requests deemed not clinically justified due to the results not being accessed or used in ongoing patient management within 24 hours. The intervention months had $5.9 \%$ of requests deemed not clinically justified due to the results not being accessed or used in ongoing patient management within 24 hours. After receiving the audit and feedback intervention, clinicians were more likely not to order inappropriate FBCs. This result was significant $t(4.6706)=0.0429, p=.05$.

Of note, the average pathology testing per admission decreased. The average pathology tests requested per admission was 13.40 from May 2013 until June 2015, whereas the intervention demonstrated an average of 11.73 pathology tests per admission. This decrease was significant $t(3.0015)=0.0057, p=.05$.

\subsection{FBC financial value of pathology test}

The cost of a FBC within this research hospital was $\$ 16.95$ (AUD) per test.

\subsubsection{Pre-intervention}

The pre-intervention data indicated that for every 10 patients receiving a FBC, 3.54 would benefit and 6.46 would not benefit from the pathology test. In financial terms, this indicates that $\$ 169.50$ would need to be spent, with an overspend of $\$ 109.50$, to benefit 3.54 patients. This trend could be categorized as low-value pathology testing. ${ }^{[13]}$

\subsubsection{Post-intervention}

The post-intervention data indicated that for every 10 patients receiving a FBC; 7.66 would benefit and 2.34 would not benefit from the pathology test. In financial terms, this indicates that $\$ 169.50$ would need to be spent, with an overspend of $\$ 39.66$, to benefit 7.66 patients. This trend could be categorized as medium-value pathology testing. ${ }^{[13]}$

This is an improvement from the pre-intervention overspend of $\$ 109.50$ (low-value) to $\$ 39.66$ (medium-value) post intervention. Due to the small sample size, the results did not directly contribute significant financial gains, although the hospital did demonstrate a reduction in the amount spent on pathology testing. Researchers compared the hospital's financial data from July, August, and September 2014 to the intervention periods financial data. Results demonstrated a

ISSN 1927-6990 E-ISSN 1927-7008 
significant reduction $t(5.7750)=0.0045, p=.05$. Results reflect previous research ${ }^{[5]}$ which demonstrated significant financial impacts, by reducing inappropriate pathology.

\subsection{Framework for analysis of test ordering}

Researchers collected and compared data from the preintervention month of June to the intervention month of September. As discussed, the pre-intervention month had more FBC pathology tests deemed inappropriate, with subsequently more feedback sessions occurring, compared to the last intervention month of September.

\subsubsection{Pre-intervention framework}

The pre-intervention month had the following results $(\mathrm{N}=73)$ :

- $42(57 \%)$ could not adequately recall why they ordered the FBC, with many request forms submitted 6-24 hours in advance

- $49(67 \%)$ highlighted potential for incidental, nonsignificant findings

- $59(81 \%)$ highlighted that they ordered the FBC with other "required" tests, i.e., highlighted that since they were taking blood they added a FBC as a potential time saver

- $7(9.6 \%)$ highlighted they did not realize the bloods had been taken and repeated the test

- $54(74 \%)$ acknowledged the test ordered would not alter their management

- $65(89 \%)$ highlighted potential risks of ordering or not ordering the test

- $51(70 \%)$ acknowledged there was a risk of over diagnosis (the cascade of tests following inconclusive results)

- $55(75 \%)$ acknowledged that a positive result was likely

- 47 (64\%) highlighted the prevalence of the provisional diagnosis

- $58(79 \%)$ admitted they were influenced by something else, including medico-legal reasons and other clinicians requesting certain tests

- $62(85 \%)$ recalled guidelines related to this presentation, although $52(84 \%)$ admitted to not applying evidence-based guidelines

\subsubsection{Intervention framework}

The last intervention month had the following results $(\mathrm{N}=11)$ :

- $1(9.1 \%)$ could not adequately recall why they ordered the FBC

- $3(27 \%)$ highlighted potential for incidental, nonsignificant findings

Published by Sciedu Press
- $4(36 \%)$ highlighted that they ordered the FBC with other "required" tests, i.e., highlighted that since they were taking blood they added a FBC as a potential time saver

- $0(0 \%)$ highlighted they did not realize the bloods had been taken and repeated the test

- $1(9.1 \%)$ acknowledged the test ordered would not alter their management

- $8(73 \%)$ highlighted potential risks of ordering or not ordering the test

- $1(9.1 \%)$ acknowledged there was a risk of over diagnosis (the cascade of tests following inconclusive results)

- $3(27 \%)$ acknowledged that a positive result was likely

- $10(91 \%)$ highlighted the prevalence of the provisional diagnosis

- $3(27 \%)$ admitted they were influenced by something else, including medico-legal reasons and other clinicians requesting certain tests

- $10(91 \%)$ recalled guidelines related to this presentation, although $5(45.5 \%)$ admitted to not applying evidence-based guidelines

The average comparisons between the pre-intervention and final intervention month "Framework for analysis of test ordering" was $69.55 \%$ vs. $36.30 \%$ respectively. This result was significant $t(2.5724)=0.01484, p=.05$.

\section{Discussion}

This study found that audit and feedback sessions significantly improved appropriate pathology ordering and specifically the clinical reasoning associated with FBCs. Before feedback, clinical reasoning for FBC pathology testing was poor. This study had the effect of promoting adequate clinical reasoning and note taking within the patient record and on the pathology request form. This is believed to have improved appropriate medical test ordering.

By focusing on the clinical reasoning associated with ordering FBC pathology testing, the researcher was able to enhance the tests' value per patient, by specifically reducing those tests of low-value. While the results did not directly contribute significant financial gains, the hospital did demonstrate a reduction in the amount spent on pathology testing from the previous year. The research outcomes provide a clinical example of how audit and feedback can promote high-value pathology ordering. It is believed this process could be applied to other medical tests, improving their testing value, which in turn helps provide a more sustainable healthcare system.

A key limitation of this study was the rotating nature of the 
junior medical officers, and the small sample size. On average, the interns and residents rotate clinical placement every three months. To be effective, appropriate medical test ordering will need to be reinforced to every new clinical rotation. To maintain positive results, the hospital has established regular education modules, with a specific focus of pathology test ordering, in conjunction with conducting regular audit and feedback interventions focusing on appropriate medical test ordering. Previous research ${ }^{[5]}$ has demonstrated that this strategy can "reduce inappropriate pathology test ordering, commonly over-ordered pathology test ordering, and pathology specimen error rates while maintaining positive patient outcomes". The researcher believes that continued auditing and feedback in conjunction with regular education modules will continue trends of reducing inappropriate pathology testing.

\section{CONFlicts OF INTEREST Disclosure}

The author would like to acknowledge he is an employee of Calvary Health Care.

\section{REFERENCES}

[1] Britt H, Miller G, Henderson J, et al. A decade of Australian general practice activity 2004-05 to 2013-14. Sydney: Sydney University Press; 2014.

[2] McGregor M, Martin D. Testing 1, 2, 3. Is overtesting undermining patient and system health? Canada Family Physician. 2012; 58(11): 1191-3. PMid: 23152453.

[3] Report of the second phase of the review of NHS pathology. London: Department of Health; 2008.

[4] Moynihan R, Doust J, Henry D. Preventing overdiagnosis: how to stop harming the healthy. BMJ Open. 2012; 344. http: //dx. doi .org/10.1136/bm .e3502

[5] Gardiner F. Medical stewardship: Pathology evidence based ordering to reduce inappropriate test ordering within a teaching hospital. J Hosp Adm. 2015. http://dx.doi.org/10.5430/jha.v5n1p73

[6] Encouraging quality pathology ordering in Australian's public hospitals. Canberra: National Coalition of Public Pathology; 2012.

[7] Quality use of pathology program. 2008. Available from: http://www.health.gov.au/internet/main/publishing .nsf/Content/qupp-hist-reports

[8] Lilford R, Bentham L, Armstrong M. What is the best strategy for investigating abnormal liver function tests in primary care? Implications from a prospective study. BMJ Open. 2013; 3(6): 3. PMid: 23794594. http://dx.doi.org/10.1136/bmjopen-2013-003 099

[9] Willis C, Metz M, Hiller J. Vitamin B12 and folate tests: the ongoing need to determine appropriate use and public funding. Med J Aust. 2013; 11(198): 2013-6. http://dx.doi.org/10.5694/mja12.1 1328

[10] Roti E, Gardini E, Magotti M. Are thyroid function tests too frequently and inappropriately requested? Journal Endocrinol Investment. 1999; 3(22): 184-90. PMid: 10219885. http://dx.doi.org /10.1007/BF03343539

[11] Morgan S, Coleman J. We live in testing times: Teaching rational test ordering in general practice. Australian Family Physician. 2014; 5(43): 273-6.

[12] Bayram C, Britt H, Miller G, et al. Evidence- practice gap in GP pathology test ordering: a comparsion of BEACH pathology data and recommended testing. Sydney: The University of Sydney; 2009.

[13] Owens DK, Qaseem A, Chou R, et al. High-Value, Cost-Conscious Health Care: Concepts for Clinicians to Evaluate the Benefits,
Harms, and Costs of Medical Interventions. Ann Intern Med. 2011; 154(3): 174-80. PMid: 21282697. http://dx.doi.org/10.7326 /0003-4819-154-3-201102010-00007

[14] Sood R, Sood A, Ghosh A. Non-evidence-based variables affecting physicians' test-ordering tendencies: a systematic review. Netherlands Journal of Medicine. 2007; 65(5): 167-77. PMid: 17519512.

[15] Van Bokhoven M, Pleunis-van Empel M, Koch H, et al. Why do patients want to have their blood tested?A qualitative study of patient expectations in general practice. BMC Family Practice. 2006; 7(2): 75. PMid: 17166263. http://dx.doi.org/10.1186/1471-229 $6-7-75$

[16] Axt-Adam P, van der Wouden J, van der Does E. Influencing behavior of physicians ordering laboratory test: a literature study. Medical Care. 1993; 31(9): 784-94. PMid: 8366680. http://dx.doi.org /10.1097/00005650-199309000-00003

[17] Moynihan R, Doust J, Henry D. Preventing overdiagnosis: how to stop harming the healthy. BMJ. 2012; 344(3502): 1-6. http: //dx.doi.org/10.1136/bmj.e3502

[18] Martin E. Deeble Institute for Health Policy Research: Australian Healthcare and Hospitals Association. 2015 February 12. Available from: http://apo.org.au/files/Resource/deeble_issue s_brief_no_8_martin_e_rationing_in_healthcare.pdf

[19] Graves N. Rationing for health services. In: Swan N, editor. Australian Broadcasting Corporation: RN2013.

[20] Fuchs VR. Eliminating "Waste" in Health Care. JAMA. 2009; 302(22): 2481-2. PMid: 19996406. http://dx.doi.org/10.10 $01 /$ jama.2009.1821

[21] About us: LCM Health Care. 2015 [updated May 14]. Available from: http: //www.calvary-act.com.au/about.html

[22] About ACT Pathology Canberra: ACT Government. 2015 [updated May 14]. Available from: http://health.act.gov.au/our-s ervices/act-pathology/about-act-pathology

[23] RCPA Manual Sydney: Royal College of Pathologists of Australasia. 2015 [updated February 9]. Available from: https://www.rcpa.edu.au/Library/Practising-Patholo gy/RCPA-Manual/Home

[24] May T, Clancy M, Critchfield J, et al. Reducing Unnecessary Inpatient Laboratory Testing in a Teaching Hospital. American Journal of Clinical Pathology. 2006; 2(126): 200-6. http://dx.doi .org/10. 1309/WP59YM73L6CEGX2F 